\title{
La vigilancia de la salud en España: ¿necesidades de mejora?
}

Health surveillance in Spain: needs for improvement?

María Jesús Terradillos García

${ }^{1}$ Instituto de Salud Carlos III, Madrid, España.

Fechas · Dates

Recibido: 2019.09.02

Aceptado: 2019.10.24

Publicado: 2020.01.15
Correspondencia · Corresponding Author

María Jesús Terradillos García

Instituto de Salud Carlos III (ISCIII)

mjtginss@hotmail.com 
La Organización Internacional del Trabajo (OIT) define la vigilancia de la salud en el trabajo como la recopilación, el análisis, la interpretación y la difusión continuada y sistemática de datos a efectos de la prevención ${ }^{(1)}$, y comprende tanto la vigilancia de la salud de los trabajadores (vigilancia de la salud individual) como la del medio ambiente de trabajo (vigilancia de la salud colectiva), siendo las actividades de vigilancia de la salud individual fundamentalmente de carácter diagnóstico-preventivo, orientadas a la detección precoz de posibles daños derivados del trabajo, mientras que las de nivel colectivo son de carácter epidemiológico. Uno de los instrumentos de los que se vale la medicina del trabajo es precisamente esta vigilancia de la salud, que permite evaluar, controlar y realizar un seguimiento de la repercusión de las condiciones de trabajo sobre la salud de la población trabajado$\mathrm{ra}^{(2)}$. Se trata, pues, de una técnica complementaria de las utilizadas por el resto de disciplinas de prevención de riesgos laborales -seguridad, higiene y ergonomía y psicosociología- por lo que no tiene sentido su consideración como instrumento aislado de prevención: ha de integrarse en las políticas de prevención globales de la empresa.

En relación con la vigilancia de la salud individual, la propia OIT indica que, si bien los exámenes de salud juegan un importante papel en la vigilancia de la salud, no deberían llevarse a cabo de manera rutinaria, sino que habrían de atender a los principios de relevancia, especificidad y sensibilidad, revisando periódicamente los programas de vigilancia de la salud a medida que se produzcan cambios y evoluciones en las condiciones de trabajo.

En España existe un largo recorrido en la práctica de los exámenes de salud en el ámbito laboral. Han transcurrido sesenta años desde la publicación de la derogada $\mathrm{OSME}^{(3)}$ (Ordenanza de los Servicios Médicos de Empresa) que establecía la realización de reconocimientos médicos a los trabajadores al menos una vez al año incluyendo radioscopias de tórax, investigaciones de componentes anormales y de sedimento en la orina, recuento de hematíes y leucocitos, fórmula leucocitaria y velocidad de eritrosedimentación, así como un examen psicotécnico elemental de acuerdo con las condiciones requeridas para su puesto de trabajo. Estos requerimientos que tan obsoletos pueden parecer hoy en día -en contraposición con las características de la vigilancia de la salud que nuestra actual normativa dispone en la Ley de Prevención de Riesgos Laborales, indicando que deberá realizarse en función de los riesgos inherentes al trabajo y sin determinar periodicidad ni pruebas complementarias concretas ${ }^{(4)}$ - lamentablemente están más cerca de la práctica actual de la vigilancia de la salud de lo que cabría esperarse.

En numerosas empresas sigue siendo muy habitual en nuestros días el ofrecer un examen médico anual a todos sus trabajadores, independientemente del puesto de trabajo desempeñado y de los riesgos laborales a los que están expuestos, dejando de lado la especificidad que debería imperar en la vigilancia de la salud. En la mayoría de las ocasiones se trata de acuerdos recogidos en los convenios colectivos de las empresas, interpretándose como mejora en la vigilancia de la salud de los trabajadores, excediendo, sin embargo, el ámbito laboral. Es posible que a esta situación contribuyan determinados factores históricos, sociales y cul- 
turales, como entender el reconocimiento médico de empresa como un "derecho adquirido" por parte tanto de los trabajadores como de los agentes sociales, así como algo "tangible" para el empresario, que lo interpreta como una contrapartida a la inversión realizada en prevención, o como un modo de cumplir con los requisitos legales ${ }^{(5)}$.

El sentir generalizado de los profesionales sanitarios en salud laboral es que la práctica de la medicina y enfermería del trabajo se ve reducida a la vigilancia individual de la salud(6), limitándose a su vez esta vigilancia de la salud a los exámenes médicos que, por otra parte, no se atienen al principio de especificidad establecido en la normativa vigente ${ }^{(7)}$, siendo más bien exámenes de salud general que laboral. Esta supremacía de la vigilancia de la salud individual tiene como consecuencia la escasa o inexistente posibilidad de llevar a cabo actividades de vigilancia de la salud colectiva, a pesar de que el RD 843/2011 determina que la dedicación a dicha actividad no deberá ser inferior a un tercio del tiempo de trabajo(8).

Otro de los problemas a los que se enfrentan en no pocas ocasiones los médicos y enfermeros del trabajo es a la falta de información de exposición laboral a la hora de establecer un protocolo de vigilancia de la salud, circunstancia ésta especialmente preocupante, ya que sin ella la medicina y enfermería del trabajo pierden todo su sentido.

Es interesante mencionar que la literatura científica es cada vez más crítica con la realización de exámenes de salud rutinarios, previos e iniciales, así como con las valoraciones de aptitud para el puesto de forma habitual, recomendando limitarlas a puestos de trabajo de alto riesgo. En el caso de algunos de los países de nuestro entorno, como Francia, la vigilancia de la salud individual se lleva a cabo de modo altamente específico en función de los riesgos laborales inherentes a cada puesto (Loi relative au travail, à la modernisation du dialogue social et à la sécurisation des parcours professionnels. L. No 2016-1088 y Décret relatif à la modernisation de la médecine du travail. D. No 2016-1908), y reservando la valoración de la aptitud únicamente para riesgos muy concretos (amianto, plomo, agentes cancerígenos, entre otros). En Alemania, el empresario sólo tendrá que poner una vigilancia de la salud individual a disposición de los trabajadores si en la evaluación de riesgos se determina que éstos están expuestos a riesgos relacionados con su puesto de trabajo y tareas desempeñadas (Arbeitsschutzgesetz, 7 Ago 1996, y Verordnung über die Fürsorge für die Gesundheit bei der Arbeit,18 Dic 2008).

Esta dedicación menos exhausta a los exámenes médicos permite al médico y enfermero del trabajo la realización de actividades colectivas en el seno de la empresa, como los estudios de puesto de trabajo, el conocimiento y evaluación del medio laboral, las campañas de promoción de la salud y de formación e información a los trabajadores, aspectos esenciales para la consecución de una vigilancia de la salud efectiva.

Cabe mencionar, además, que las previsiones futuras respecto al número de especialistas en medicina del trabajo indican un déficit de profesionales ${ }^{(9)}$, por lo que se impone la optimización de los recursos disponibles y la adecuada planificación de las actividades preventivas. 
Así pues, conviene plantearse si la práctica actual de los profesionales en salud laboral que dedican gran parte de su tiempo a realizar exámenes de salud de carácter genérico debe mantenerse, en detrimento de una vigilancia de carácter colectivo y de tareas de investigación, tan necesarias ante las nuevas patologías que están surgiendo relacionadas con los cambios organizativos y tecnológicos a los que estamos asistiendo en los últimos años. Analizando la normativa vigente en materia de prevención de riesgos laborales, bastaría con la observancia de lo estipulado a la hora de organizar la actividad preventiva para dotar de una mayor relevancia a la vigilancia de la salud colectiva, si bien será necesaria una labor de educación y concienciación previa dirigida tanto a empresarios como a trabajadores, para evitar que este cambio pudiera ser interpretado como una pérdida de derechos laborales. No debe olvidarse que el objetivo de la vigilancia de la salud en el trabajo es el de detectar aquellos factores que estén incidiendo negativamente en la salud de los trabajadores para poder establecer las medidas que los eliminen; ello refuerza aún más la filosofía de los exámenes de salud dirigidos a circunstancias laborales concretas, y no a condicionantes generales de salud.

Pronto van a cumplirse 25 años desde la promulgación de la Ley de Prevención de Prevención de Riesgos Laborales y, a pesar de haberse avanzado notablemente en este terreno, todavía queda camino por recorrer. Debemos aunar esfuerzos para optimizar y mejorar la calidad de los sistemas de vigilancia de la salud mediante la utilización de herramientas de medicina basada en la evidencia, de modo que se cumplan los criterios de especificidad, validez científica y eficacia. Por otra parte, deben facilitarse los medios para la realización de estudios epidemiológicos y para la investigación en salud laboral que permita continuar avanzando en la mejora de la salud y de las condiciones de trabajo de nuestra sociedad.

\section{Bibliografía}

1. Organización Internacional del Trabajo. Technical and ethical guidelines for workers' health surveillance. Ginebra: OIT; 1998.

2. Solé MD, Solórzano M, Piqué T. Nota técnica de prevención 959: La vigilancia de la salud en la normativa de prevención de riesgos laborales. Madrid: Instituto Nacional de Seguridad e Higiene en el Trabajo; 2012.

3. Orden por la que se aprueba el Reglamento de los Servicios Médicos de Empresa. TIN/2504/2010 (21 Nov 1959).

4. Ley de Prevención de Riesgos Laborales. L. No 31/1995 (8 Nov 1995).

5. Rodríguez-Jareño M, Molinero E, de Montserrat J, Vallès A, Aymerich M. Calidad y adecuación técnica a la normativa de los exámenes de vigilancia de la salud de la población trabajadora en relación con su finalidad preventiva. Arch Prev Riesgos Labor. 2016;19:146-65.10..

6. Rodríguez-Jareño MC, de Montserrat i Nonó J. ¿Es posible mejorar la utilidad preventiva de la vigilancia de la salud de los trabajadores en el actual marco normativo? Arch Prev Riesgos Labor. 2017;20:80-101. 
7. Reglamento de los Servicios de Prevención. RD N 37/1997 (17 Ene 1997).

8. Real Decreto por el que se establecen los criterios básicos sobre la organización de recursos para desarrollar la actividad sanitaria de los servicios de prevención. RD No 843/2011 (17 Jun 2011).

9. Barber P, González B. Estimación de la oferta y demanda de médicos especialistas. España 2018-2030. 2018 [citado 26 Dic 2019]. Disponible en: https:// www.mscbs.gob.es/profesionales/formacion/necesidadEspecialistas/doc/20182030EstimacionOfertaDemandaMedicosEspecialistasV2.pdf 